Das Parlament kann zurzeit die Weichen stellen für eine gesündere Schweiz: in beiden Kammern stehen gleichzeitig die Gesetzgebungen zu Alkohol, Tabakerzeugnissen und zum Glücksspiel auf der Tagesordnung. Die gesundheitlichen Folgeschäden von Nikotin, übermässigem Alkoholkonsum und ruinösem Glücksspiel sind bekannt. Der Gesetzgeber ist hier gefordert, mit einer kohärenten Suchtpolitik der Prävention in allen drei Gebieten nachdrücklich Geltung zu verschaffen und dafür zu sorgen, dass Süchtige Rahmenbedingung vorfinden, die ihnen den Zugang zu ihren Suchtmitteln erschweren und den Zugang zu Hilfe erleichtern.

Dr. med. Christine Romann, Mitglied des Zentralvorstandes der FMH, Departementsverantwortliche Gesundheitsförderung und Prävention

\title{
Einmalige Chance für kohärente Suchtpolitik
}

\section{Barbara Weil}

Leiterin Abteilung Gesundheitsförderung und Prävention

Drogen und Suchtverhalten sind eine Realität. Der Wunsch nach einer suchtfreien Gesellschaft darf nicht verhindern, dass man sich pragmatisch mit der Wirklichkeit auseinandersetzt. Wie in anderen Ländern führt die Suchtpolitik auch in der Schweiz zu zahlreichen Kontroversen: Die Vielfalt der Ansichten drückt sich in unterschiedlichen Wertvorstellungen und Standpunkten aus, die man bei der Ausarbeitung von Lösungsvorschlägen geltend machen kann. Ar-

\section{Sucht ist eine Realität, die vor allem auch die Gesellschaft und Umwelt angeht.}

gumente, die zu sehr auf Emotionen, Moral oder Idealismus gründen, verdecken dabei den Blick auf die Realität und berücksichtigen insbesondere die oft verzweifelte Lage der abhängigen Menschen nicht.

Ärztinnen und Ärzte sind bei ihrer Tätigkeit täglich mit Suchtproblemen konfrontiert. Die Therapie und weitere Hilfestellungen werden durch das soziale und rechtliche Umfeld beeinflusst. Abhängigkeit ist eine Krankheit wie jede andere auch: Betroffene Personen benötigen somit primär Hilfe.

Nach wie vor ist es wichtig, das Problem illegaler Drogen im Zusammenhang aller psychoaktiver Substanzen zu sehen, die zu einer Abhängigkeit führen können wie Alkohol, Tabak oder auch psychotrope Medikamente. In jüngster Zeit ist auch eine Zunahme von sogenanntem nicht substanzgebundenem Suchtverhalten zu verzeichnen wie etwa Internetsucht oder Glücksspielsucht.

$\mathrm{Zu}$ Beginn jeder Abhängigkeit steht die Früherkennung - sie stellt somit die wichtigste Funktion des Arztes in der Prävention dar. Eine systematische Anamnese des Konsums ermöglicht ein Eingreifen, bevor es zu einer schweren Abhängigkeit oder zu Kom- plikationen kommt. Besonders bei Jugendlichen und jungen Erwachsenen in schwierigen Lebenssituationen empfiehlt es sich, das Thema direkt und offen anzusprechen.

Daneben kann das Suchtverhalten entscheidend beeinflusst werden durch rechtliche Rahmenbedingungen, welche Verfügbarkeit und Zugang limitieren: Im Internet kann rund um die Uhr gespielt werden, die Schweiz hat eine der grössten Casinodichten weltweit, Alkohol ist rund um die Uhr erhältlich.

Seit der Veröffentlichung des ersten Drogenkonzepts der FMH im Jahr 1996 hat sich an diesen Tatsachen wenig geändert. Aktuell haben wir jedoch in der Politik erneut die Gelegenheit, eine glaubwürdige Suchtpolitik zu ermöglichen: Beide Parlamentskammern prüfen gleichzeitig Gesetzgebungen zu Alkohol, Tabakerzeugnissen und Glücksspiel; Anfragen von Kantonen und Städten zur Cannabisfragen kommen hinzu.

Eine kohärente Sucht- und Präventionspolitik macht nur Sinn, wenn nicht die Inhalte jedes dieser drei Gesetze von Interessengruppen so zerpflückt werden, als hätten sie keinen Zusammenhang - wenn nicht um jeden Präventions-Franken, um jede strukturelle Präventionsmassnahme gekämpft werden muss, deren Wirksamkeit längst nachgewiesen ist.

\section{Der Markt beeinflusst das Verhalten von} Individuen - besonders von vulnerablen Menschen.

Die FMH fordert eine umfassende realistische Sichtweise und präventionspolitische Massnahmen, die aufeinander abgestimmt und nicht gegenläufig sind; Suchtverhalten ist nicht nur ein individuelles Problem - sondern ein gesamtgesellschaftliches. 